\title{
Machine Vision System Based on the Parallel Shift Technology and Multiple Image Analysis
}

\author{
Stepan Belan ${ }^{1} \&$ Sergey Yuzhakov ${ }^{1}$ \\ ${ }^{1}$ State Economic and Technologies Transport University, Kiev, Ukraine \\ Correspondence: Stepan Belan, State Economic and Technologies Transport University, Kiev, Lukashevicha str., \\ 19, 03049, Ukraine. Tel: 380-445-915-127. E-mail: bstepan@ukr.net
}

Received: July 15, 2013 Accepted: September 13, 2013 Online Published: September 28, 2013

doi:10.5539/cis.v6n4p115 URL: http://dx.doi.org/10.5539/cis.v6n4p115

\begin{abstract}
This article describes basic methods that are required to build a system of machine vision which based on parallel shift technology. The methods are based on the implementation of one operation (definition function of the area of intersection of the real image and its copy which parallel shifted) and analysis of its basic characteristics. Analysis of time intervals obtained characteristics improves the performance of image recognition. The methods are based on simple mathematical operations and can be used in the processing of both raster and non-raster images.
\end{abstract}

Keywords: image recognition, function of the area of intersection, parallel shift, image noise, image area, movement parameters, spatial orientation, contour, vision system

\section{Introduction}

Construction system of machine vision requires the creation of effective methods for determining movement parameters of objects, their spatial orientation and edge detection.

In modern methods performing these operations is provided by compulsory conversion of images into digital form (Gonzalez, Woods, \& Eddins, 2004; Chen, Rau, \& Wang, 1995). Further processing of video data occurs by performing of various mathematical operations on set of pixels. Sometimes mathematical transformation of the data can be quite difficult. Obviously, the real biological systems of vision are operating otherwise.

There is a need to put in the basis of systems of vision simple methods of operation with video data (Belan \& Yuzhakov, 2002, 2008, 2013; Belan \& Belan, 2012; Belan \& Motornyuk, 2013). Such methods of image processing can be done using parallel shift technology (PST). This name proposed by the authors and has not been used in the scientific literature. PST involves converting some static events in dynamic characteristics with help forced changes the input object.

In this case, a static image is converted to a set of functions by interacting with its copy. It is based on a simple circuit technique solutions and mathematical operations (definition of area, shift and comparison). Also in this case does not provided compulsory conversion of images to digital form. Modeling of all processes related to the image processing by using parallel shift technology, the authors conducted by using bitmaps.

For the analysis basic characteristics of the image at the input of machine vision proposed approach which based on the representation (description) image by one of characteristics. This characteristic is a function of the area of intersection (FAI) of image and its copy that parallel shifted in the appropriate direction (Belan \& Yuzhakov, 2013). It is served as a graph, and analysis of its basic components allows not only to recognize an image, but also to determine movement parameters of the object, its spatial orientation, detection of contour, etc.

This article reflects the possibility of solving the basic problems of video information processing by using homogeneous parameter (area). The mathematical models of basic image processing operations using FAI and their parameters constructed. Proposed methods provide a basis for building a machine vision system based on PST.

Currently there are no video systems that allow providing the implementation of these methods. There is a need creation for specialized video cameras that would ensure the realization of cyclic shifts for the process obtaining of FAI. Using this method of image conversion has advantages in speed of processing video only when FAI has 
building by hardware.

\section{Determination of the Basic Parameters of Moving Objects in the Visual Scene by Analyzing the Function of the Area of Intersection}

In the plan of identifying and detection of basic parameters moving objects in video surveillance systems developed by a number of methods and tools (Belan \& Motornyuk, 2013; Niitsuma \& Maruyama, 2005; Talukder \& Maithies, 2004; Lu, Wang, Wu, \& Yang, 2008; Doğan, Serhan, \& Sitk1, 2010; Yuan \& Hanspeter, 2010). In these works the implementation of calculations is different and conducted by a comparison of neighboring frames of video stream. In many implementations performed on personal computers and programmable logic device. That is, they have not reached the full parallelism of computing, which affects performance.

Process and mathematical model obtaining of FAI for image recognition has been described in a previous article (Belan \& Yuzhakov, 2013). PST also allows you to effectively determine the movement parameters of the object with high speed. The main features include movement direction, distance and speed of the displacement of the object.

To determine the movement parameters of the object displacement its copies should be carried out cyclically. That is, when copy of the object reached the edge of receptive field in the direction of displacement, this copy has to appear at the same level on the other hand receptive field and continue to shift in the original direction (Figure 1.a). The painted area is corresponds the function of the area of intersection (FAI) for the displacement $x_{i}$. Then the function of the area of intersection will be periodic and symmetrical about each point of complete coincidence of input image if it does not move, and its copy that moves (Figure 1.b). In this figure FAI shown schematically.

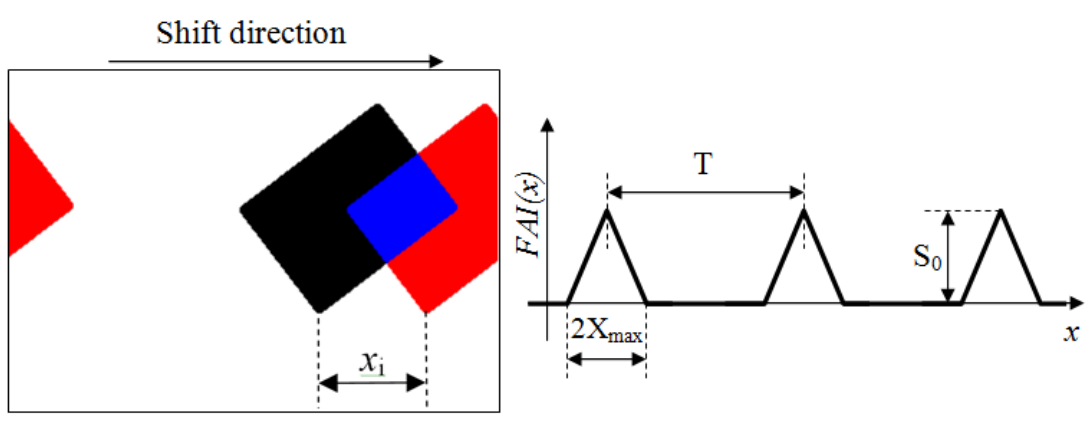

a)

b)

Figure 1. Cyclic shift to determine the movement parameters of the object

This parameters of function of the area of intersection will be equal to the following values: period (T) of FAI the width of the receptor field at horizontal shift and height at vertical shift, the width of each peak FAI (part functions corresponding to nonzero intersection) $-2 \mathrm{X}_{\max }$ or $2 \mathrm{Y}_{\max }$ in accordance the direction of displacement, the maximum amplitude of FAI - input image area $\left(\mathrm{S}_{0}\right)$. We had chosen displacement in orthogonal directions because of the ease of hardware implementation of this process. Theoretically, you can choose any two directions of displacement. Period $(\mathrm{T})$ is calculated in units of distance measurement. This is convenient for processing raster images. For non raster images may be convenient to express this parameter due to the speed shift of image copy $(\mathrm{V})$ and time $(\mathrm{t})$, for which occurs a certain process. For example

$$
T=V t_{T} \text { or } X_{\max }=V t_{X_{\max }}
$$

This method is similar to the method of determining the parameters of binary stars and planets in astronomy that is based on the analysis of cyclic brightness functions. For static images is introduced forced cyclic shift of the copy. The main factor in the creation of systems for determining the movement parameters is movement speed of the image copy. 
If the object is moving in the receptor field in the orthogonal direction, then the cyclic shift in the same direction period (T) will vary depending on the direction of the shift of figure. At movement in the direction of displacement of copy period between the peaks of function (call it $T_{1}$ ) due to the Doppler effect will be greater than $\mathrm{T}$, and at movement in the opposite direction less.

If the movement the image is not orthogonal, provided that the copy of is shifting so fast that by the time of maximum intersection the object shifted not much in the direction perpendicular to the displacement of copy, then each peak will be symmetric, but its amplitude less than at immovable object. Then the period of function is defined as the distance between the midpoints of two adjacent peaks with maximum amplitude.

Assume that a copy of the figures is moving at determining the $F A I(x)$ to the right, and at determining the $F A I(y)$ - upwards and center of mass of the object is at the point of beginning coordinates. Then the tangent of the angle at the direction of motion the image $(\alpha)$, if the movement is not orthogonal, will be:

$$
\operatorname{tg} \alpha=\frac{T_{y 1}-T_{y}}{T_{x 1}-T_{x}}
$$

where $\mathrm{T}_{\mathrm{y} 1}-$ period $F A I(y)$ at the moving object, $\mathrm{T}_{\mathrm{y}}-$ period $F A I(y)$ at the immovable object, $\mathrm{T}_{\mathrm{x} 1}$ and $\mathrm{T}_{\mathrm{x}}-$ corresponding periods $F A I(x)$.

Determination period $F A I(y)$ of the immovable object $\left(\mathrm{T}_{\mathrm{y}}\right)$ is equal to the height of receptive field. Period $\mathrm{T}_{\mathrm{x}}$ is equal to the width of the receptive field.

At orthogonal movements of the object:

$$
\Delta T_{y}=T_{y 1}-T_{y}=0
$$

if the object is moving horizontally;

$$
\Delta T_{x}=T_{x 1}-T_{x}=0
$$

if the object is moving vertically.

Signs above differences and $\operatorname{tg} \alpha$ uniquely determine the movement direction of the object relatively its previously location (Figure 2), and absolute differences - the distances of the displacement in that direction.

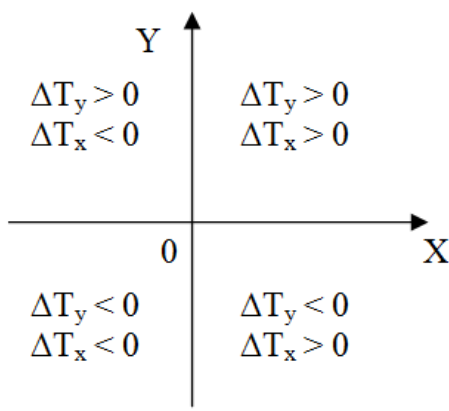

Figure 2. The conditions for determining the direction of displacement

Thus, the reaction on moving and determination its basic parameters limited only the technical capabilities of realization the speed of cyclic shift.

The restrictions based on the properties of orthogonal movement can be used in video control systems for road, rail and water transport. This is because the capability maneuvering these types of transport located in a horizontal direction. Such restrictions can greatly simplify the construction of automatic systems for video control of the movement parameters of objects. It is required the creation video control systems with obtaining of FAI. Example from program which simulates the proposed method is shown in Figure 3. 


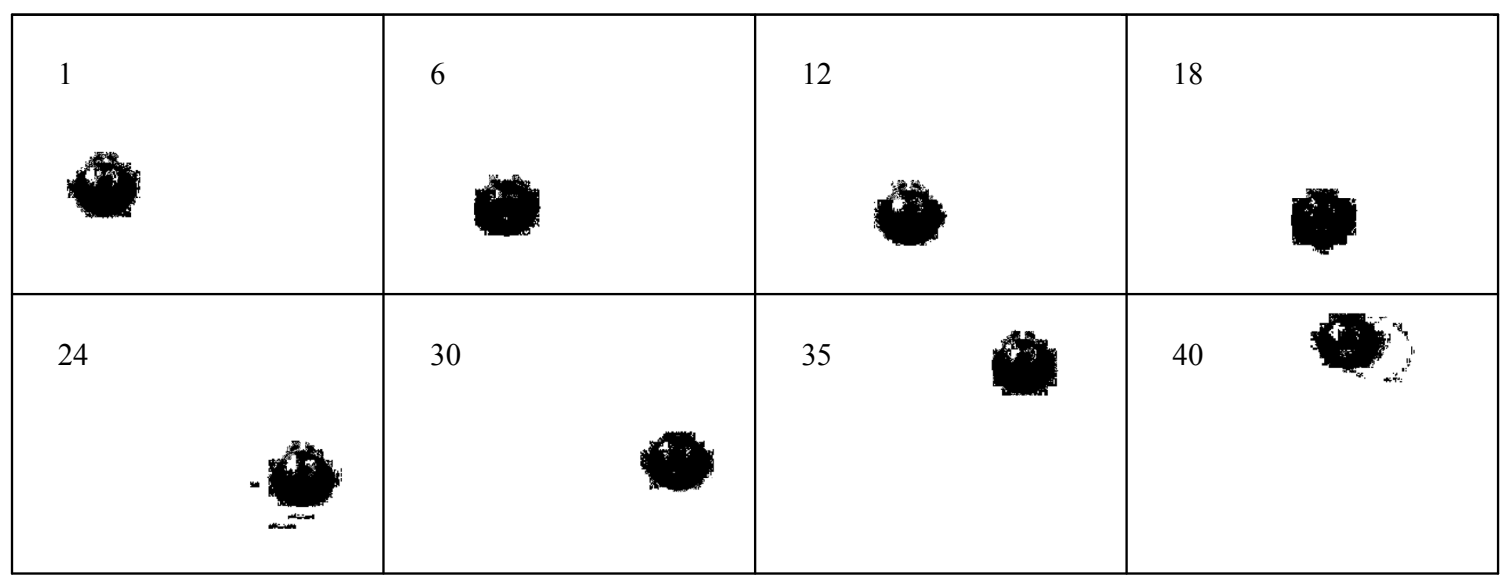

a) An example of the moving image

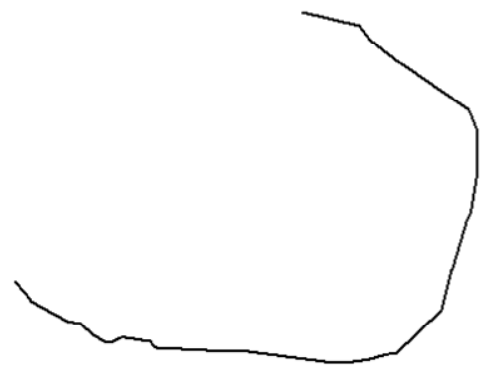

b) The trajectory obtained by observing the movement of the center of mass of the object

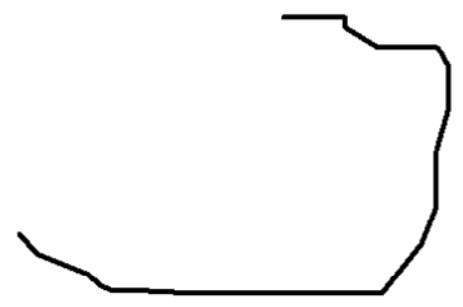

c) The trajectory obtained by the analysis of FAI

Figure 3. The trajectory of an object by using different methods for tracking the movement of the object

Proposed method for determining movement parameters can be used at a constant distance to the object. If it is approaching or is removed relative of visual system, it is necessary to carry out certain adjustments.

Determining the distance to the object can be done in many ways. For example, using ultrasonic echolocation or lasers, by construction systems of binocular vision. These methods are shown just an example and later are not used. It is proposed the method of determining the distance to an $2 \mathrm{D}$ object by using simple geometric properties. Determination of the initial position of the object can be performed using the property of similarity of figures (Figure 4).

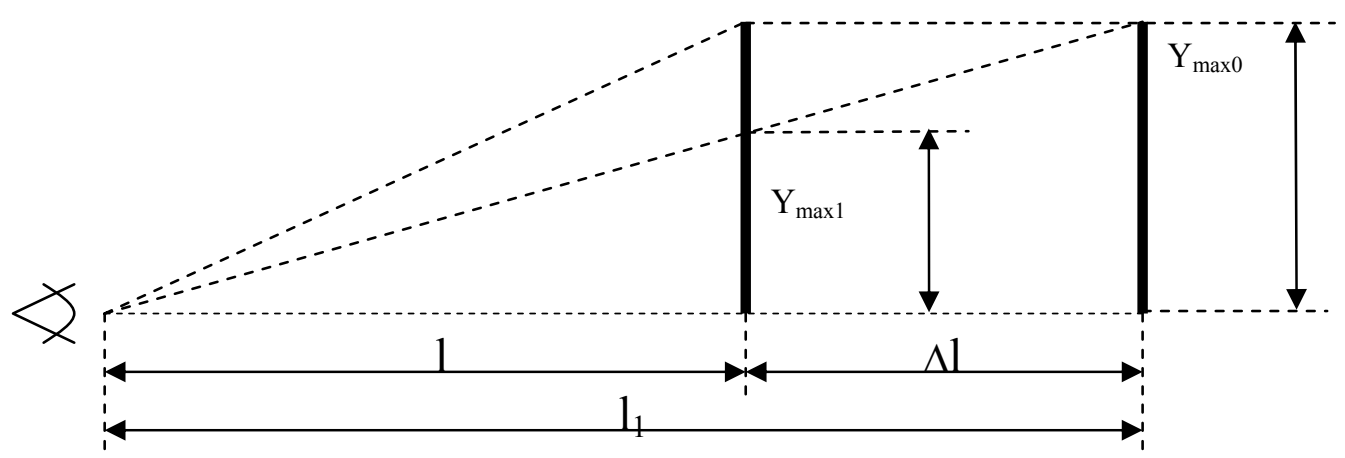

Figure 4. Determining the distance to the object

According to these properties:

$$
\frac{l+\Delta l}{l}=\frac{Y_{\max 0}}{Y_{\max 1}}=\sqrt{\frac{S_{0}}{S_{1}}}
$$


Using (4) it follows:

$$
l=\frac{\Delta l \sqrt{S_{1}}}{\sqrt{S_{0}}-\sqrt{S_{1}}}
$$

To determine the initial distance to the object we can use the changing distance by the movement of the optical system. Thus given a certain value $\Delta l=l_{1}-l$. Also, the procedure for obtaining parameter $\Delta l$ can be done by modifying the focal length of the accepting camera that must be pre-calibrated to perform such a procedure. This is analogous to the process of accommodation of the eye. For determine values $\sqrt{S_{0}}$ and $\sqrt{S_{1}}$ necessary to determine the area of the object in the moments before and after the change of focal length or the changing distance to optical system.

The initial location of the object can also be determined using parallel shift technology. The initial coordinates of figure in the plane of the visual field is defined as the distance that corresponds to the start of the shift until reaching the edge of receptive field by the middle of the object copy in appropriate direction in the first cycle of displacement.

It should be noted that this method of determining the initial distance is dependent on the accuracy of data acquisition by optical system.

\section{Method of Determining the Spatial Orientation of Images}

The PST allows effectively determine the spatial orientation of the image of object.

Suppose we have an image, whose parameters are stored in the memory of the vision system as etalon $A$. At an arbitrary location in space of the image it will appear as a projection $B$ on a plane of receptive field (Figure 5). The angle $\gamma$ is the tilt angle of the etalon $A$ to the plane of receptive field. The angle $\varphi$ indicates the direction of the line with respect to which there etalon rotates.

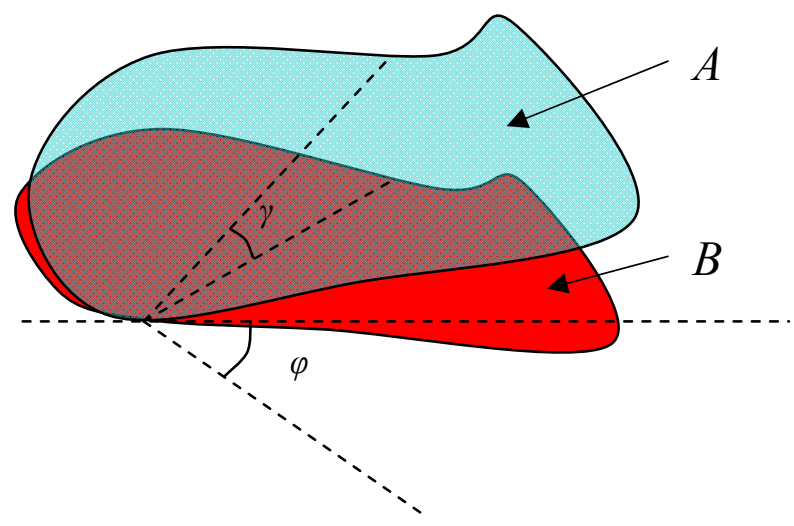

Figure 5. Projection B of etalon A for an arbitrary plane

Recall the formula integral indicator (Belan \& Yuzhakov, 2013). It has the ability to not change with scale and based on the basic parameters of the function of the area of intersection $\left(\mathrm{S}_{0}, \mathrm{X}_{\max }, \mathrm{Y}_{\max }\right)$.

$$
k_{x}=\frac{\int_{0}^{X_{\max }} F A I(x) d x}{S_{0} X_{\max }}=\text { const }
$$

where $x$ and $X_{\max }$ - single (basic) and maximal shift in $\varphi$ direction. Integral indicators of projection $B$ and of etalon $A$ in the directions $\varphi$ and $\phi+\frac{\pi}{2}$ will have the following
properties.

$$
\begin{aligned}
& k_{x_{A}}=k_{x_{B}} \\
& k_{y_{A}}=k_{y_{B}}
\end{aligned}
$$

$k_{\mathrm{x}}$ are integral indicators in the direction $\varphi$ and $k_{\mathrm{y}}$ are integral indicators in the direction $\phi+\frac{\pi}{2}$. 
This stage coincides with fast stage of algorithm of image recognition using integral indicators from the article (Belan \& Yuzhakov, 2013). Ability to determine the angle $\varphi$ is provided by preservation etalons as reference surfaces.

On detailed stage is performed comparison of scalable with preserving the proportions FAI of the real images and etalons that selected in the previous stage for each step of the shift. There may be four variants of the results of the comparison.

Variant 1.

$$
\frac{F A I(x)_{B}}{F A I(x)_{A}}=\text { const }=\cos \gamma
$$

Then it requires the comparison of scalable functions in the direction $\phi+\frac{\pi}{2}$.

If

$$
\frac{F A I(y)_{B}}{F A I(y)_{A}}=\text { const }>1
$$

Then this etalon is selected correctly. The projection is relative to the direction $\varphi$.

Otherwise we must move to another etalon for which true formulas (7) and (8).

Variant 2.

$$
\frac{F A I(x)_{B}}{F A I(x)_{A}}=\text { const }>1
$$

Then it requires the comparison of scalable functions in the direction $\phi+\frac{\pi}{2}$.

If

$$
\frac{F A I(y)_{B}}{F A I(y)_{A}}=\text { const }=\cos \gamma
$$

Then this etalon is selected correctly. The projection is relative to the direction $\phi+\frac{\pi}{2}$.

Otherwise we must move to another etalon for which true Formulas (7) and (8).

Variant 3.

$$
\frac{F A I(x)_{B}}{F A I(x)_{A}}=\text { const }=1
$$

and

$$
\frac{F A I(y)_{B}}{F A I(y)_{A}}=\text { const }=1
$$

Then this etalon is selected correctly. The shape of real image $B$ is fully consistent with the shape of chosen etalon $A$.

Otherwise we must move to another etalon for which true Formulas (7) and (8).

Variant 4. If

$$
\frac{F A I(x)_{B}}{F A I(x)_{A}} \neq \text { const }
$$

or

$$
\frac{F A I(y)_{B}}{F A I(y)_{A}} \neq \text { const }
$$

Then this etalon is selected not correctly. We must move to another etalon for which true Formulas (7) and (8).

If the comparison does not match any etalon, for which the real image $(B)$ is the projection, the system should provide learning process to create the new etalon $(B)$. 
We can accurately determine the spatial orientation of the $2 \mathrm{D}$ image relatively of the plane of receptor field if the parameters available among an array of etalons by calculated with this method the angles $\varphi$ and $\gamma$. This method can be used to ensure the functioning of systems "eye-hand" autonomous robots and on conveyor lines.

\section{Method of Contour Definition of Plane Figures}

Definition the contour of images is one of the main directions of research of machine vision systems. While a PST should be used primarily for the not contour objects processing, its use will can determine the contour of an image. Currently there are many contour detection methods which used practically in all existing means of processing and pattern recognition. This is due to the fact that the contour is $80 \%$ information of the image object (Belan, 2011; Kozhemyako, Bilan, \& Savaliuk, 1997).

To apply the proposed method, we assume let the contour of the figures is a layer of given thickness $(h)$, which belongs to the figure and is at its external borders. To determine the contour for a given technology at cyclic shift copies of images carried out the count down intervals $t_{y}-t_{y+h}$ and $t_{y-h}-t_{y}\left(t_{x}-t_{x+h}\right.$ and $\left.t_{x-h}-t_{x}\right)$, where $t_{y}\left(t_{x}\right)-t$ point of maximum amplitude of the $F A I(y)(F A I(x))$, and $\mathrm{t}_{\mathrm{y}-\mathrm{h}}, \mathrm{t}_{\mathrm{y}+\mathrm{h}}, \mathrm{t}_{\mathrm{x}-\mathrm{h}}, \mathrm{t}_{\mathrm{x}+\mathrm{h}}-$ times required to shift the copies of object to a distance $h$. Like contour points we name the points of the object that are not included in the intersection with a copy at the shift to the distance $h$ (black color pixels on Figure $6 . \mathrm{b} \div \mathrm{e}$ ). The contour is defined as a superposition of contour areas that defined by horizontal and vertical displacements (Figure 6.f).

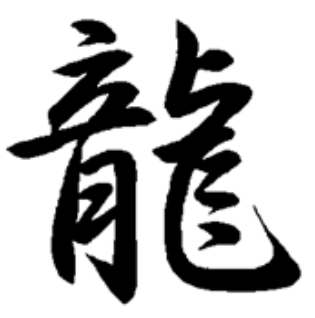

a) initial image

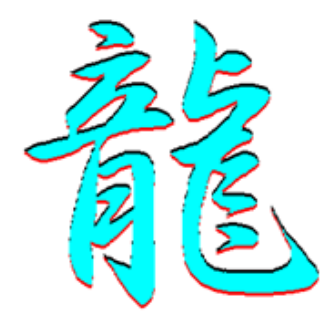

d) down shift

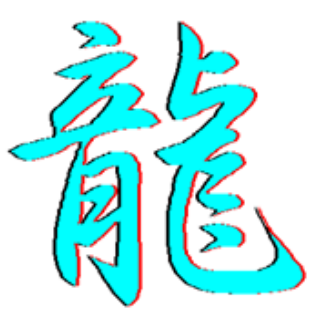

b) right shift

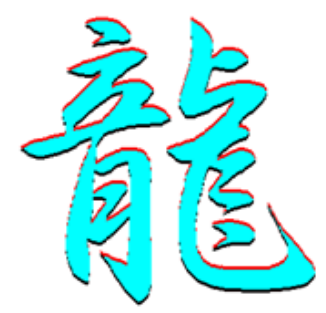

e) up shift

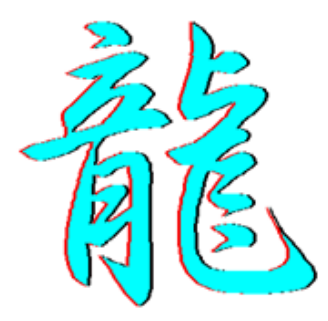

c) left shift

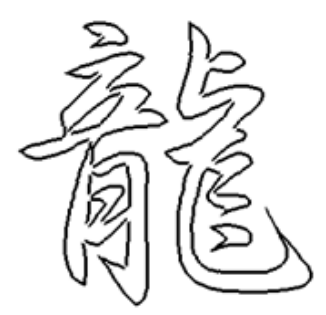

f) contour

Figure 6. Process of edge detection with $h=3$ pixels

The process of edge detection can also be used for noise control. In this case, all parts of the image that belong to the contour must be removed. In the simulation program used the type of noise "salt" and "pepper".

Method deletes groups of small elements that do not belong to image. But plots of the white elements of noise at the image increase their size by the removing the elements that surround them. Deleted the elements edge of the image must be considered when determining the quantitative parameters of the object (Figure 7.a,b). These negative factors of using this type of noise control. 


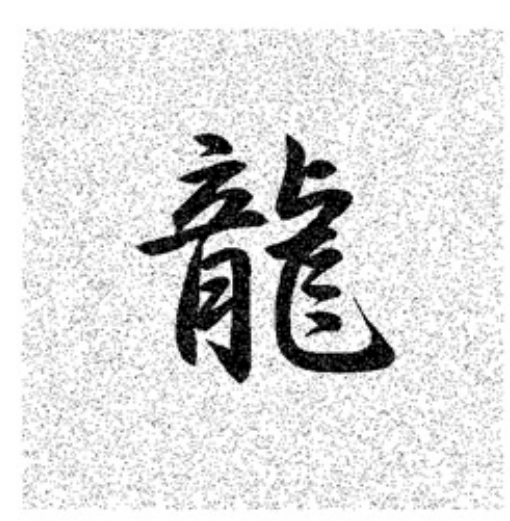

a) initial image at PSNR $=34 \mathrm{~dB}$ and type of noise "salt" and "pepper"

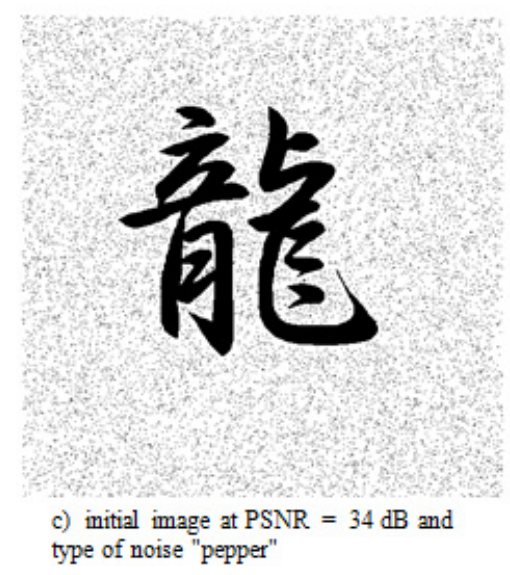

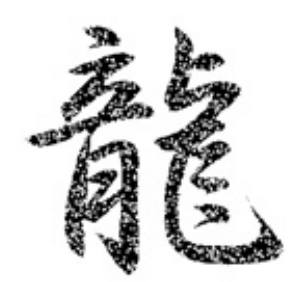

b) after noise control at $h=1$ pixel

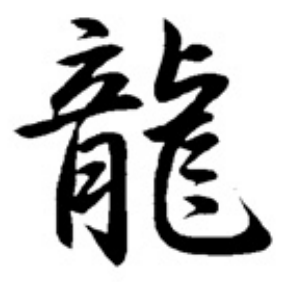

d) after noise control at $h=1$ pixel

Figure 7. Using edge detection for noise control

The advantage is the fact that this method is entirely based on the parallel shift technology. It does not require the use of any other methods and can be used when preprocessing is not bitmaps. In addition, when you use the types of noises that do not distort the object, the method of noise control significantly increases its efficiency (Figure 7.c,d).

\section{Machine Vision Systems Based on Multiple Image Analysis}

From the above mentioned facts it is clear that there is able to build the system of machine vision which based on the parallel shift technology. This system can recognize images for which etalons exist, determine the basic parameters of movement of the object, determine the spatial orientation of the image relative of the plane of receptive field, determine the distance to the object, find the contour, delete noise.

The basis of this technology is receiving cyclic functions of the area of intersection. For this purpose, on the two layers of matrix of cyclic shift registers that reflect the receptor field, simultaneously in both directions are performed orthogonal cyclic shifts of the input image copies to obtain $F A I(x)$ and $F A I(y)$. Each of the plots obtained $F A I$ is responsible for ability of a machine vision system that has been described above (Figure 8).

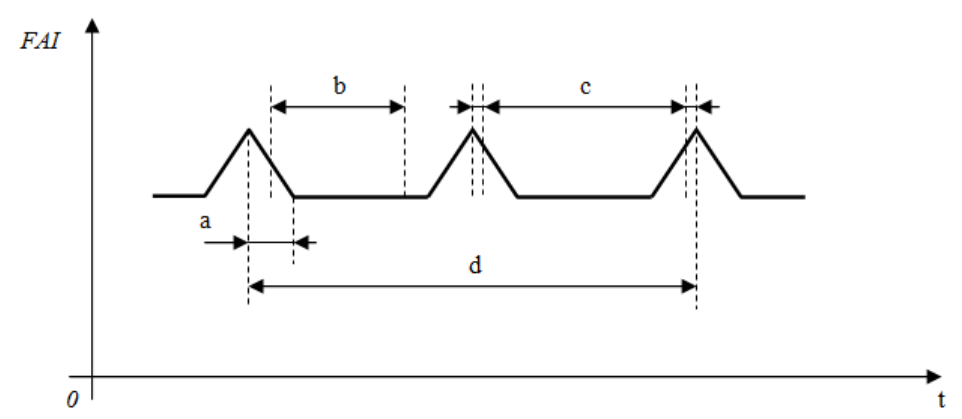

Figure 8. The distribution of FAI plots to perform certain operations 
Plot $a$ is used for image recognition, and in the process of determining spatial orientation, plot $b$ - in determining the initial location coordinates, plots $c$ - in determining the contour of the image and in process of noise control, plot $d$ - in determining the movement parameters of the object. Chart of FAI (Figure 8) shown schematically and only for one direction of displacement. Right boundary of plot $b$ is chosen arbitrarily. It depends on the real distance from the object to the edge of the receptive field. Specialization of plots FAI for other direction is similar. It represented dependence of the $F A I$ on the time $(\mathrm{t})$. To determine the dependence FAI on the values of distance parameter enough corresponding periods of time multiply by the speed of the shift of copy. Schema of the machine vision system shown in Figure 9.

Machine vision system which based on parallel shift technology can quite accurately simulate the vision of living creatures. It may explain why the pupil of the eye are moving in image recognition and defining the contours of figures. The method of calculating the basic movement parameters of the object can explain the processes going on in the experiments to determine the properties of the eyes of creatures respond to the motion. Changing the focal length of the camera to determine the distance to an object similar to the process of accommodation of the eye. Ability to determine the angles of the plane, which belongs figure simplifies the operation of the system "eye-hand".

Conversion an image to the set of functions of the area of intersection makes it easy to integrate this system with the human nervous system. It can be used in prosthetics eye in medicine. In this case, in the system "sensor-block analysis" of the role of the sensor will perform a device obtaining of FAI, and the human brain will be the block of analysis.

The simplicity and multifunctionality of system which based on parallel shift technology will be used in devices vision of autonomous robots. As you know, simplicity - is the basis of reliability. Reliability is especially needed in cases where not possible to repair the system operatively.

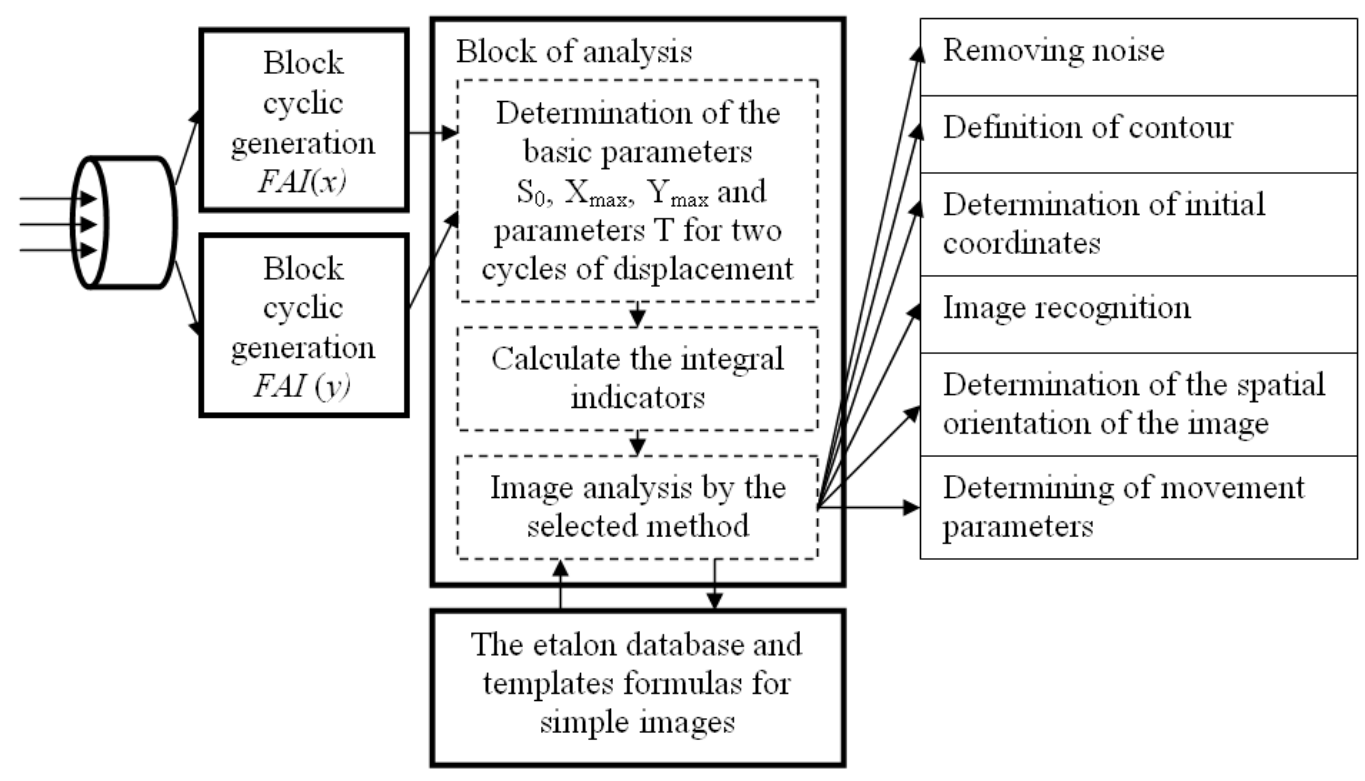

Figure 9. Schema of the machine vision system

Manipulating with functions and the ability to process non-raster images can be used in analog computers.

Maybe there are better methods to implement these functions. But this technology is self sufficient. It will allow in the complex to realize basic video processing tasks without using other technologies.

\section{Conclusion}

The method of definition of movement parameters, spatial orientation and detection contour of the object together with the image recognition are form the basis of any machine vision system. The parallel shift technology and multiple image analysis greatly reduces the time defining the basic parameters of images that recognized. This is because for all operations of processing and description of the images is determined by only one characteristic. The parallel shift technology is based on a simple operations (determining the area, shift, 
comparison), so its methods easy to implement in hardware. Using these methods possible when processing both raster and not bitmaps.

Machine vision system based on parallel shift technology can be used in transport, medicine, at the construction of autonomous robots and conveyor systems for manufacturing.

Further is necessary to research image recognition only by a variety of indicators which independent of the scale (integral indicators, etc.). It will allow to use tools of the theory of solutions for recognition. Is necessary explore the possibility of constructing neural network, for which as the input vector is array of integral indicators of image. Need to research also of the methods of analysis scenes and binocular machine vision system based on the parallel shift technology. It requires a hardware tools construction of cyclic FAI.

\section{References}

Belan, S. (2011). Specialized cellular structures for image contour analysis. Cybernetics and Systems Analysis, 47(5), 695-704. http://dx.doi.org/10.1007/s10559-011-9349-8

Belan, S., \& Belan, N. (2012). Use of Cellular Automata to Create an Artificial System of Image Classification and Recognition. In Cellular Automata (pp. 483-493). Springer Berlin Heidelberg. http://dx.doi.org/10.1007/978-3-642-33350-7_50

Belan, S. N., \& Motornyuk, R. L. (2013). Extraction of characteristic features of images with the help of the radon transform and its hardware implementation in terms of cellular automata. Cybernetics and Systems Analysis, 49(1), 7-14. http://dx.doi.org/10.1007/s10559-013-9479-2

Belan, S., \& Yuzhakov, S. (2002). Recognition of plane figures based on analysis of the area of intersection of similar objects. Herald VPI, 3, 74-78.

Belan, S., \& Yuzhakov, S. (2008). Method of image recognition on the basis of the process of parallel shear for systems of object recognition at railway crossings. Collection of scientific works of the State Economic and Technologies Transport University, 13, 216-226.

Belan, S., \& Yuzhakov, S. (2013). A Homogenous Parameter Set for Image Recognition Based on Area. Computer and Information Science, 6(2), 93-102. http://dx.doi.org/10.5539/cis.v6n2p93

Chen, C. H. (2009). Handbook of pattern recognition and computer vision. World Scientific.

Chunrong, Y., \& Hanspeter, A. (2010). Mallot Real-Time Detection of Moving Obstacles from Mobile Platforms. In ICRA10. Workshop on Robotics and Intelligent Transportation System (pp. 109-113).

Doğan, S., Temiz, M. S., \& Külür, S. (2010). Real time speed estimation of moving vehicles from side view images from an uncalibrated video camera. Sensors, 10(5), 4805-4824. http://dx.doi.org/10.3390/s100504805

Gonzalez, R. C., Woods, R. E., \& Eddins, S. L. (2004). Digital Image Processing using MATLAB.

Kozhemyako, V. P., Belan, S., \& Savaliuk, I. (1997, February). Optoelectronic self-regulation neural system for treatment of vision information. In International Conference on Optical Storage (pp. 120-126). International Society for Optics and Photonics.

Lu, N., Wang, J., Wu, Q. H., \& Yang, L. (2008). An improved motion detection method for real-time surveillance. IAENG International Journal of Computer Science, 35(1), 1-10.

Niitsuma, H., \& Maruyama, T. (2005, August). Real-time generation of three-dimensional motion fields. In Field Programmable Logic and Applications, 2005. International Conference on (pp. 179-184). IEEE.

Talukder, A., \& Matthies, L. (2004, October). Real-time detection of moving objects from moving vehicles using dense stereo and optical flow. In Intelligent Robots and Systems, 2004. (IROS 2004). Proceedings. 2004 IEEE/RSJ International Conference on (Vol. 4, pp. 3718-3725). IEEE.

\section{Copyrights}

Copyright for this article is retained by the author(s), with first publication rights granted to the journal.

This is an open-access article distributed under the terms and conditions of the Creative Commons Attribution license (http://creativecommons.org/licenses/by/3.0/). 